\title{
A Normalized Approach for Designing Cylindrical Microresonators
}

\author{
Dion J. W. Klunder, Member, IEEE, H. J. W. M. Hoekstra, and Alfred Driessen, Senior Member, IEEE
}

\begin{abstract}
A normalized design approach has been developed that allows rapid estimates of parameters like the bend loss, finesse, and free spectral range of the fundamental microresonator modes of two- and three-dimensional microresonators. Based on this approach, the minimum size for a given finesse and the free spectral range for microresonators in silicon oxynitride technology are investigated.
\end{abstract}

Index Terms-Cylindrical microresonators, normalized analysis, optical waveguides, waveguide theory.

\section{INTRODUCTION}

A COMMON approach for designing waveguide-coupled cylindrical microresonators (MRs) is to describe their response by an analytical model with the propagation losses and roundtrip phase shifts of the MR-modes and their coupling with the modes of the adjacent straight waveguides (SWs) as input parameters (see, for example, [1]-[3]). In most cases, one is primarily interested in the on-/off-resonance behavior of the MR and as a consequence, the round-trip phase shift is considered as a given parameter. A more detailed analysis [2], [3] shows that the attainable ranges for parameters like, e.g., the finesse or the intracavity power, are determined by the propagation losses of the MR-modes and can be varied within the available range by controlling the coupling between the $\mathrm{SW}$ - and MR-modes. It is therefore crucial to determine the propagation losses of the MR-modes in order to obtain a first estimate of the performance of a MR.

The propagation losses are the sum of the inherent bend losses, which are determined by the refractive indexes and the geometry of the MR, of the surface roughness induced scattering losses and the materials losses. The magnitudes of the materials and scattering losses depend on the materials and the quality of the layers and the etching processes, respectively, and can be reduced - for a given material—by optimization of the technology. For given refractive indexes and geometry, the bend losses are a fixed parameter and set an inherent lower limit for the propagation losses of the MR-modes. Accurate calculations of the bend losses require rigorous mode solvers for bend

Manuscript received October 11, 2002; revised January 29, 2003. This work was supported by the Nederlandse Organizatie voor Wetenschappelijk Onderzoek (NOW) under the Research Program of Stichting voor Fundamenteel Onderzoek der Materie (FOM).

D. J. W. Klunder was with the Lightwave Devices Group, MESA+ Research Institute and Department of Applied Physics, University of Twente, $7500 \mathrm{AE}$ Enschede, The Netherlands. He is now with C2V, 7500 AH Enschede, The Netherlands.

H. J. W. M. Hoekstra and A. Driessen are with the Lightwave Devices Group, MESA+ Research Institute and Department of Applied Physics, University of Twente, 7500 AE Enschede, The Netherlands.

Digital Object Identifier 10.1109/JLT.2003.810567 waveguides [4], [5] and are time-consuming. Moreover, the calculated bend losses are specific for a given geometry, and the calculations should (in principle) be repeated for different geometries. Normalized curves for the bend losses of bends with one-dimensional (1-D) cross-sections are proposed by [6] and [7], where bend losses are calculated with a relative accuracy $<20 \%$ for moderate ratios between the refractive index of the core $\left(\mathrm{n}_{\text {core }}\right)$ and the index of the background $\left(\mathrm{n}_{\mathrm{bg}}\right)$ layers $\left(\mathrm{n}_{\text {core }} / \mathrm{n}_{\mathrm{bg}} \leq 1.2\right)$. This approach can considerably reduce the design effort, but seems to be less applicable for larger ratios between the index of the core and background layers.

In this paper, a normalized approach for designing two-dimensional (2-D) and three-dimensional (3-D) cylindrical microresonators is presented. The approach is based on reducing the 3-D MR to a 2-D MR by applying the effective index method (EIM) and using normalized graphs for the bend losses and important parameters like the finesse and free spectral range (FSR) of the fundamental microresonator modes. The accuracy of the approach is not limited by the (rigorous) normalization procedure, but by the accuracy of the finite-difference mode solver used for calculating the bend losses [5] and the EIM.

The paper is organized as follows. In Section II, starting from Maxwell's equations, normalized expressions for the bend losses, free spectral range, and the unloaded finesse of a MR are derived. Thereafter, a normalized design approach for 2-D cylindrical microresonators is presented. Based on the normalized graphs presented in Section III, a normalized design approach for 3-D MRs is presented in Section IV. Section V shows how the presented design approach can be used for estimating the minimum size for 3-D MRs with specified acceptable bend losses and given refractive indexes.

\section{SCALING OF MAXWELL'S EQUATIONS AND THE IMPLICATIONS FOR CURVED WAVEGUIDE SYSTEMS}

Maxwell's equations for linear, isotropic, source-free systems (and assuming a time-dependence $\exp (\mathrm{j} \omega \mathrm{t})$ ) are [8]

$$
\begin{array}{ll}
\text { (i) } \nabla \times \mathbf{E}=-\mathrm{j} \omega \mu_{0} \mathbf{H} & \text { (iii) } \nabla \cdot\left(\mathrm{n}^{2} \mathbf{E}\right)=0 \\
\text { (ii) } \nabla \times \mathbf{H}=\mathrm{j} \omega \varepsilon_{0} \mathrm{n}^{2} \mathbf{E} & \text { (iv) } \nabla \cdot \mathbf{H}=0
\end{array}
$$

with $\omega$ as the angular frequency, $n$ as the refractive index, $\varepsilon_{0}$ as the permittivity in vacuum, and $\mu_{0}$ as the permeability in vacuum. From (1), it is possible to derive the full-vectorial wave equations (see, e.g. [9])

$$
\begin{array}{r}
\nabla^{2} \mathbf{H}+\left(\nabla \ln \left(\mathrm{n}^{2}\right)\right) \times \nabla \times \mathbf{H}+\mathrm{k}_{0}^{2} \mathrm{n}^{2} \mathbf{H}=0 \\
\nabla^{2} \mathbf{E}+\nabla\left(\mathbf{E} \cdot \nabla \ln \left(\mathrm{n}^{2}\right)\right)+\mathrm{k}_{0}^{2} \mathrm{n}^{2} \mathbf{E}=0 .
\end{array}
$$


Equation (2) is invariant under the following set of transformations:

$$
\begin{aligned}
\mathbf{r}^{\prime} & =\frac{\mathbf{r}}{\lambda_{\text {ref }}} \\
\mathrm{n}^{\prime} & =\frac{\mathrm{n}}{\mathrm{n}_{\text {ref }}} \\
\lambda_{\text {ref }} & \equiv \frac{\lambda}{\mathrm{n}_{\text {ref }}}
\end{aligned}
$$

with $n_{\text {ref }}$ a reference index, $\mathbf{r}^{\prime}$ the position $\mathbf{r}$ normalized by the reference wavelength $\lambda_{\text {ref }}$, and $\mathrm{n}^{\prime}$ the normalized refractive index. By inserting (3) into (2), we find normalized full-vectorial wave equations

$$
\begin{array}{r}
\nabla^{\prime 2} \mathbf{H}+\left(\nabla^{\prime} \ln \left(\mathrm{n}^{\prime 2}\right)\right) \times \nabla^{\prime} \times \mathbf{H}+\left(2 \pi \mathrm{n}^{\prime}\right)^{2} \mathbf{H}=0 \\
\nabla^{\prime 2} \mathbf{E}+\nabla^{\prime}\left(\mathbf{E} \cdot \nabla^{\prime} \ln \left(\mathrm{n}^{\prime 2}\right)\right)+\left(2 \pi \mathrm{n}^{\prime}\right)^{2} \mathbf{E}=0
\end{array}
$$

with $\nabla^{\prime}$ corresponding to differentiation with respect to the normalized coordinate $\mathbf{r}^{\prime}$.

For a straight waveguide (SW) whose modes have propagation constants $\beta$, we find, in addition to the transformations in (3), that the modal field equations are also invariant under transforming $\beta$ to a normalized propagation constant $\beta^{\prime}$

$$
\beta^{\prime}=\beta \lambda_{\text {ref }}=2 \pi \mathrm{n}_{\text {ref }}^{\prime} \Rightarrow \mathrm{n}_{\text {eff }}^{\prime}=\frac{\mathrm{n}_{\text {eff }}}{\mathrm{n}_{\mathrm{ref}}}
$$

with $\mathrm{n}_{\mathrm{eff}}^{\prime}$ defined as the normalized effective index of the straight waveguide mode.

The angular dependence of quasi-guided modal fields in a curved waveguide, with the radius of curvature $R$, can be expressed as

$$
\{\mathbf{E}, \mathbf{H}\} \propto \exp (-\mathrm{j} \gamma \phi)
$$

with angular propagation constant

$$
\gamma=\mathrm{k}_{0} \mathrm{Rn}_{\mathrm{eff}} .
$$

By inserting (6) into the full-vectorial wave equations for curved waveguides (4), it can be shown that the resulting modal field equations and the angular propagation constants $\gamma$ are invariant under transformations (3)

$$
\begin{aligned}
\mathrm{r}^{\prime} & =\frac{\mathrm{r}}{\lambda_{\text {ref }}} \\
\mathrm{y}^{\prime} & =\frac{\mathrm{y}}{\lambda_{\text {ref }}} \\
\phi^{\prime} & =\phi \\
R^{\prime} & =\frac{R}{\lambda_{\text {ref }}} \\
\mathrm{n}^{\prime} & =\frac{\mathrm{n}}{\mathrm{n}_{\text {ref }}} \\
\gamma^{\prime} & =\gamma \\
\mathrm{n}_{\text {eff }}^{\prime} & =\frac{\mathrm{n}_{\text {eff }}}{\mathrm{n}_{\text {ref }}}
\end{aligned}
$$

with $r, y$, and $\phi$ as the cylindrical coordinates defined in Fig. 1.

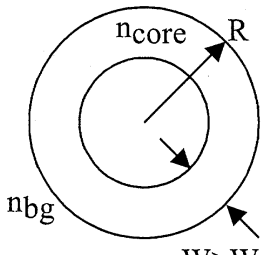

a)

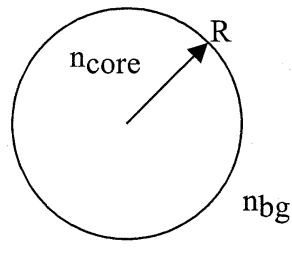

b)
Fig. 1. (a) A 2-D micro ring resonator and (b) micro disk resonator with core index $\mathbf{n}_{\text {core }}$ and background index $\mathbf{n}_{\mathrm{bg}}$ with $\hat{\mathbf{r}}, \hat{\phi}$, and $\hat{\mathbf{y}}$ as the unit vectors of the cylindrical coordinate system and $R$ and $W$ as the radius of curvature and ring width, respectively.

As a consequence of the invariance of the angular propagation constant, the round-trip losses $\left(\alpha_{\mathrm{rtrp}}\right)$ and effective indexes $\left(\mathrm{n}_{\mathrm{eff}}\right)$ of the microresonator modes are invariant as well

$$
\begin{aligned}
\operatorname{Re}\left\{\gamma^{\prime}\right\} & =\operatorname{Re}\{\gamma\}=\frac{2 \pi \mathrm{Rn}_{\mathrm{eff}}}{\lambda}=2 \pi R^{\prime} \mathrm{n}_{\mathrm{eff}}^{\prime} \Rightarrow \mathrm{n}_{\mathrm{ef}}^{\prime}=\frac{\mathrm{n}_{\mathrm{eff}}}{\mathrm{n}_{\mathrm{ref}}} \\
\alpha_{\mathrm{rtrp}}^{\prime} & =\alpha_{\mathrm{rtrp}}=-54.575 \times \operatorname{Im}(\gamma),[\mathrm{dB}] .
\end{aligned}
$$

The finesse of an unloaded MR (i.e., no coupling with adjacent waveguides) [3]

$$
\begin{gathered}
F^{(0)}=\frac{\pi}{2}\left\{\arcsin \left(\frac{1-\left|\mathrm{X}^{(0)}\right|}{2 \sqrt{\left|\mathrm{X}^{(0)}\right|}}\right)\right\}^{-1} \\
\text { with: }\left|\mathrm{X}^{(0)}\right|=10^{\frac{-\alpha_{\mathrm{rtrp}}}{20}}
\end{gathered}
$$

is an upper limit for the finesse. It is directly related to the round-trip losses via the fraction of the field remaining in the MR after a single roundtrip $\left(\left|\mathrm{X}^{(0)}\right|\right)$ and decreases monotonically with increasing roundtrip losses; see also Fig. 2. As a consequence, $F^{(0)}$ is also invariant under transformations (3). Approximating the real part of $\gamma$ as a first-order Taylor series results in an expression for FSR / $\lambda$ that also accounts for the effects of waveguide and materials dispersion [3]

$$
\frac{\mathrm{FSR}}{\lambda}=\left(\frac{\mathrm{FSR}}{\lambda}\right)^{\prime}=\left(\frac{1}{\operatorname{Re}\left\{\gamma^{\prime}\right\}}\right) \cdot\left(1-\frac{\lambda_{\mathrm{ref}}}{\mathrm{n}_{\mathrm{eff}}^{\prime}} \frac{\partial \mathrm{n}_{\mathrm{eff}}^{\prime}}{\partial \lambda_{\mathrm{ref}}^{\prime}}\right)^{-1} .
$$

It appears that the ratio FSR $/ \lambda$ is invariant under transformations (3). To obtain a simplified expression for FSR $/ \lambda$, one may ignore the effects of waveguide and materials dispersion (i.e., $\partial \mathrm{n}_{\text {eff }}^{\prime} / \partial \lambda_{\text {ref }}^{\prime}=0$ )

$$
\frac{\mathrm{FSR}}{\lambda}=\left(\frac{\mathrm{FSR}}{\lambda}\right)^{\prime}=\frac{1}{\operatorname{Re}\left\{\gamma^{\prime}\right\}} .
$$

It should however be remarked that for high index contrast devices, the waveguide dispersion can be quite strong [10], and as a consequence, (11) overestimates FSR / $\lambda$ by typically $20 \%$. But for estimation of the normalized FSR, (11) is rather useful, as it does not require knowledge of the wavelength dependence of the effective index. We therefore decided to use (11) in the remainder of this paper. 


\section{NORMALIZED DESIGN APPROACH FOR 2-D MICRORESONATORS}

In this section, the normalized analysis developed in the previous section is applied to the analysis of the fundamental TM-polarized modes of a cylindrical microresonators with 1-D cross-sections. In reality (see, e.g., [2]), the propagation losses of the microresonator modes also depend on the surface roughness induced scattering losses and the material losses. To keep the discussion as general as possible, the scattering and materials losses are excluded from the analysis, as they depend on specific fabrication details. If an estimate is possible, they should be added to the intrinsic bend losses resulting in an effective round-trip loss. In this way, the reduction in the unloaded finesse can easily be determined.

As an example, consider the 2-D micro ring resonator in Fig. 1(a), with ring width $W$. From (7), it follows that the modal fields of the micro ring resonator can be completely described by a set of three parameters: $W^{\prime}, R^{\prime}$ and $\mathrm{n}_{\text {core }} / \mathrm{n}_{\mathrm{bg}}$. Suppose one would like to design a micro ring resonator with an unloaded finesse $F^{(0)} \geq 500$, with a normalized FSR, FSR $/ \lambda \geq 1 \cdot 10^{-2}$ and $n_{\text {core }} / n_{\text {bg }} \leq 1.4$. From Fig. 2 , it follows that this requires losses per round-trip smaller than $\alpha_{\text {rtrp }} \leq 0.05 \mathrm{~dB}$.

In [6], it has been shown that for sufficiently large ring widths ( $W \geq W_{\mathrm{WGM}}$ ), the modes in the micro ring can be approximated by the whispering gallery modes (WGMs) of a micro disk, with $W_{\text {WGM }}$ given by

$$
\begin{aligned}
\frac{W_{\mathrm{WGM}}^{\prime}}{R^{\prime}} & =1-\left(\frac{\mathrm{n}_{\mathrm{eff}}^{\prime}}{\mathrm{n}_{\text {core }}^{\prime}}\right) \sqrt{1-\left(\frac{9}{\operatorname{Re}\left\{\gamma^{\prime}\right\}}\right)^{\frac{2}{3}}} \\
W_{\mathrm{WGM}}^{\prime} & =\frac{W_{\mathrm{WGM}}}{\lambda_{\mathrm{bg}}} \\
R^{\prime} & =\frac{R}{\lambda_{\mathrm{bg}}} \\
\lambda_{\mathrm{bg}} & =\lambda \cdot\left(\mathrm{n}_{\mathrm{bg}}\right)^{-1} \\
\mathrm{n}_{\text {core }}^{\prime} & =\frac{\mathrm{n}_{\mathrm{core}}}{\mathrm{n}_{\mathrm{bg}}} .
\end{aligned}
$$

In the remainder of this paper, we restrict the analysis to micro ring resonators whose fundamental modes are in the (so-called) WGM-regime: $W \geq W_{\mathrm{WGM}}$. As a consequence, the propagation of the fundamental mode can be described in terms of only two normalized parameters: $\mathrm{n}_{\text {core }} / \mathrm{n}_{\mathrm{bg}}$ and $R^{\prime}$.

In most practical cases, one is interested in an upper limit for the bend losses in the microresonator, and it is sufficient to consider the bend losses of the TM-polarized modes in the 2-D micro disk (modes with a nonzero electrical field in the radial direction), as they are in general larger than the bend losses of the TE-polarized mode (for the same normalized parameters). Fig. 3 shows the bend losses-obtained by using a rigorous finite-difference (FD) mode solver for bends with 1-D cross-sections [4], [5]-of the TM0 mode per round-trip in the micro disk $\left(\alpha_{\mathrm{rtrp}}\right)$ as a function of the normalized radius $R^{\prime}$ and the index ratio between the core and background $\left(\mathrm{n}_{\text {core }} / \mathrm{n}_{\mathrm{bg}}\right)$ as parameter. It should be remarked that the results

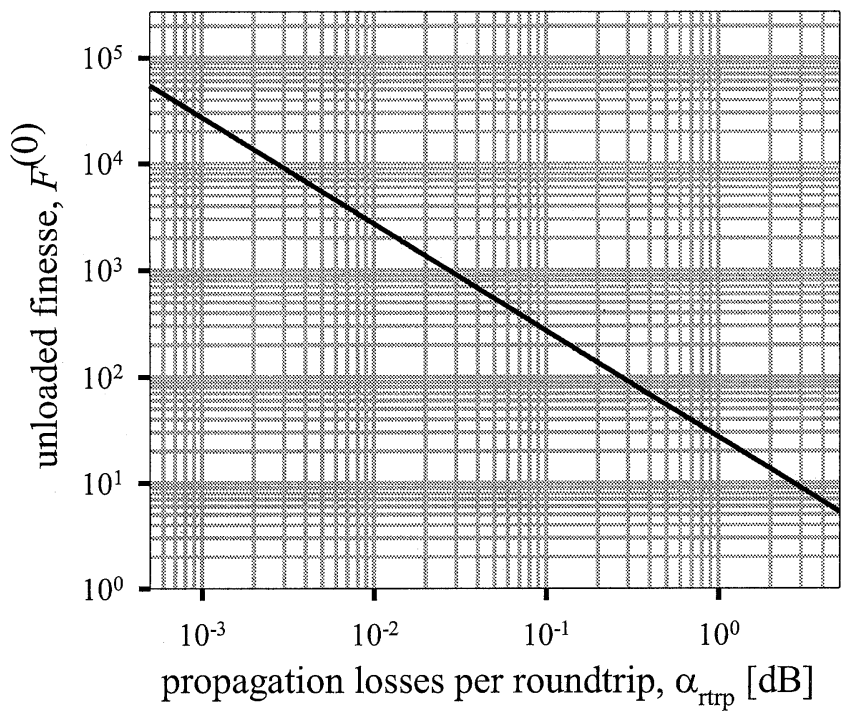

Fig. 2. Unloaded finesse — as defined in (9)—of a microresonator as a function of the propagation losses per round-trip.

for MR-modes with losses per round-trip $\alpha_{\text {rtrp }}<10^{-4} \mathrm{~dB} / 2 \pi$ have been omitted as they are close to the (double) precision used in the calculations. The results for MR-modes with high losses have been omitted as well, because their accuracy is limited by the performance of the perfectly matched layer (PML) boundary conditions [11]. Fig. 3 shows the expected behavior, namely, a monotonic decrease of the round-trip bend losses with increasing $R^{\prime}$ and $\mathrm{n}_{\text {core }} / \mathrm{n}_{\mathrm{bg}}$.

In Fig. 4, the normalized effective index as a function of $R^{\prime}$ and $n_{\text {core }} / n_{\text {bg }}$ is given (full lines). It shows that the normalized effective index increases monotonically with increasing normalized radius and the normalized index of the core. The dashed lines in Fig. 4 indicate the normalized effective index as a function of $R^{\prime}$ for given FSR $/ \lambda$. As the FSR-for fixed radius of curvature-is inversely proportional to the effective index, it can be understood that the dashed lines in Fig. 4 indicate the upper limits for the normalized effective index for a given minimum $\mathrm{FSR} / \lambda$.

From Figs. 3 and 4, it is possible to determine the ranges of normalized parameters for a given minimum FSR and unloaded finesse. As an example: it follows from Fig. 4 that $\mathrm{FSR} / \lambda \leq 1 \cdot 10^{-2}$ requires a maximum normalized bend radius of $R^{\prime}<11$ for $\mathrm{n}_{\text {core }} / \mathrm{n}_{\mathrm{bg}}=1.4$ and $\mathrm{n}_{\text {core }} / \mathrm{n}_{\mathrm{bg}}=1.05$ for $R^{\prime}<16$, respectively. For an unloaded finesse $F^{(0)} \geq 500$ (i.e., $\alpha_{\text {rtrp }} \leq 0.05 \mathrm{~dB}$ ) and $R^{\prime}=16$, it follows from Fig. 3 that $\mathrm{n}_{\text {core }} / \mathrm{n}_{\mathrm{bg}} \geq 1.2$. As a consequence, assuming no scattering and materials losses, an index ratio between the core and background of $\mathrm{n}_{\text {core }} / \mathrm{n}_{\mathrm{bg}} \geq 1.2$ and a normalized radius of curvature $R^{\prime}=11-16$ (depending somewhat on $\mathrm{n}_{\text {core }} / \mathrm{n}_{\mathrm{bg}}$ ) are required for an unloaded finesse $F^{(0)} \geq 500$ and FSR $/ \lambda \geq 1 \cdot 10^{-2}$.

It should be remarked that the accuracy of the normalized curves presented in this section is not determined by the normalization procedure but by the accuracy of the FD mode solver; for the grid size we used in our calculations, we estimated the relative error in the calculated (complex) propagation constants to be better than $1 \%$. 


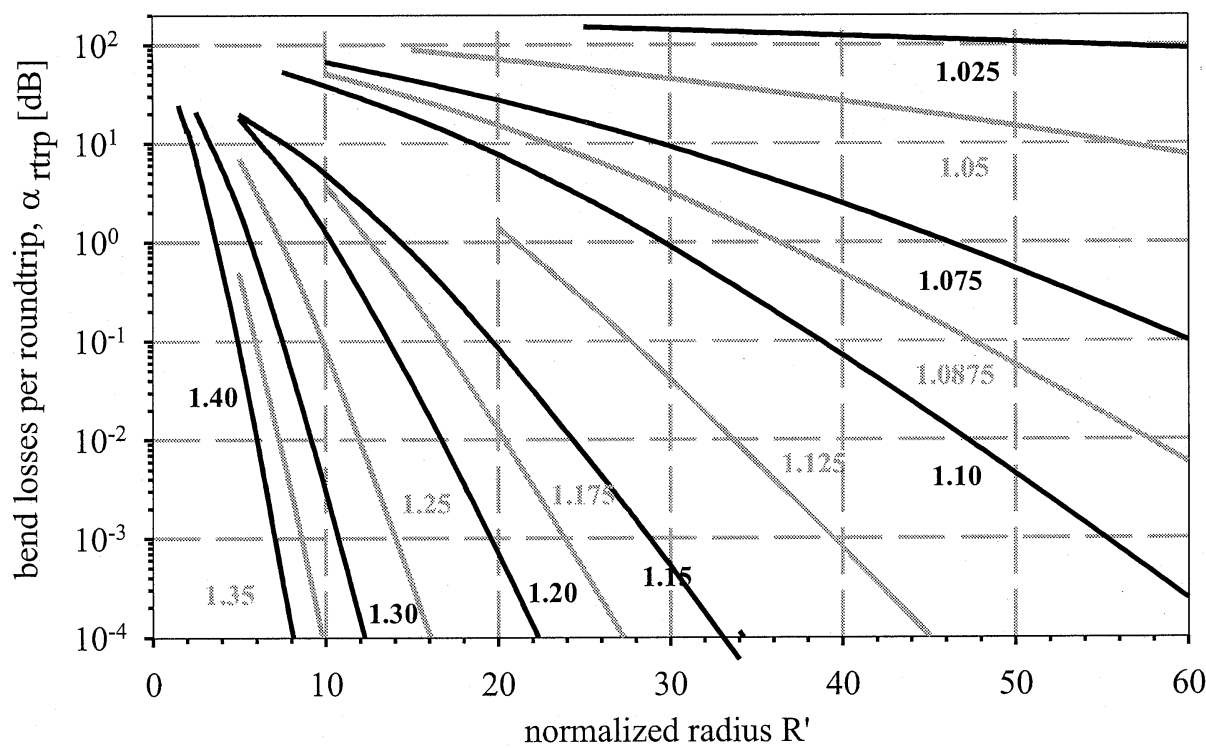

Fig. 3. Bend losses of the TM0 mode per roundtrip (on a log-scale) in the 2-D micro disk resonator as a function of the normalized radius with the ratio between the core and background index $\left(\mathbf{n}_{\text {core }} / \mathbf{n}_{\mathrm{bg}}\right)$ as parameter.

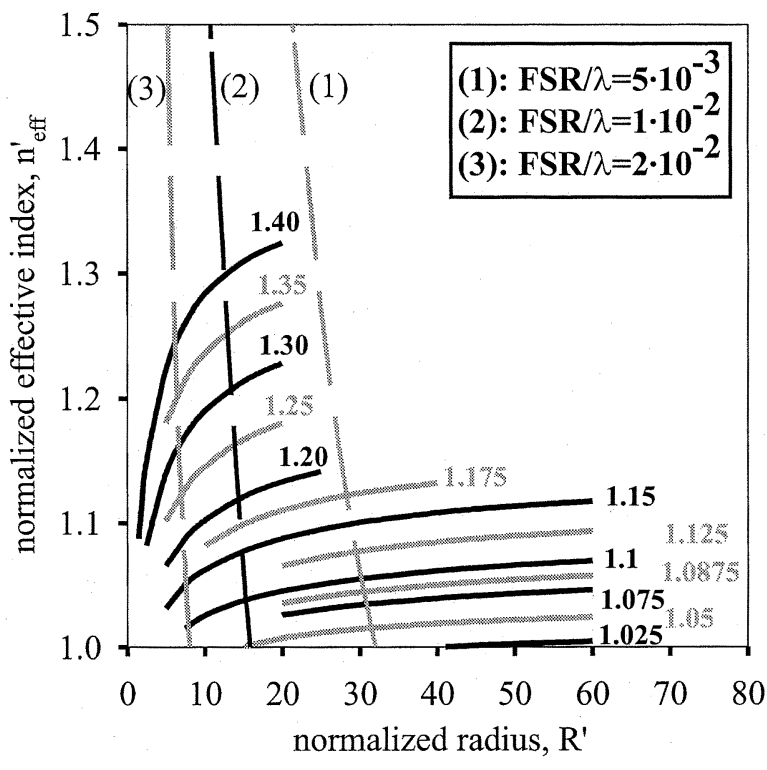

Fig. 4. Full lines: Normalized effective index—as defined in (7)—of the TM0 mode of a 2-D micro disk resonator as a function of the normalized radius and background index $\left(\mathbf{n}_{\text {core }} / \mathbf{n}_{\mathrm{bg}}=1.025-1.50\right)$ as a parameter. Dashed lines: Normalized effective index of the TM0 mode of a 2-D micro disk resonator as a function of the normalized radius and the normalized free spectral range FSR $/ \lambda$ [see (11)] as parameter.

\section{NORMALIZED DESIGN APPROACH FOR 3-D MICRORESONATORS}

In this section, it will be demonstrated how the results of the previous section can be used in the design of 3-D microresonators. The method is applied, but certainly not limited, to 3-D micro ring resonators in a silicon oxynitride materials system [12]. The thus-obtained results are compared with the results obtained by applying a rigorous 3-D FD mode solver for bends [5].

Consider the vertical cross-section in Fig. 5(a) of a micro ring resonator defined in a three-layer system. In order to apply the graphs for 2-D MRs from Section III, one first has to reduce the
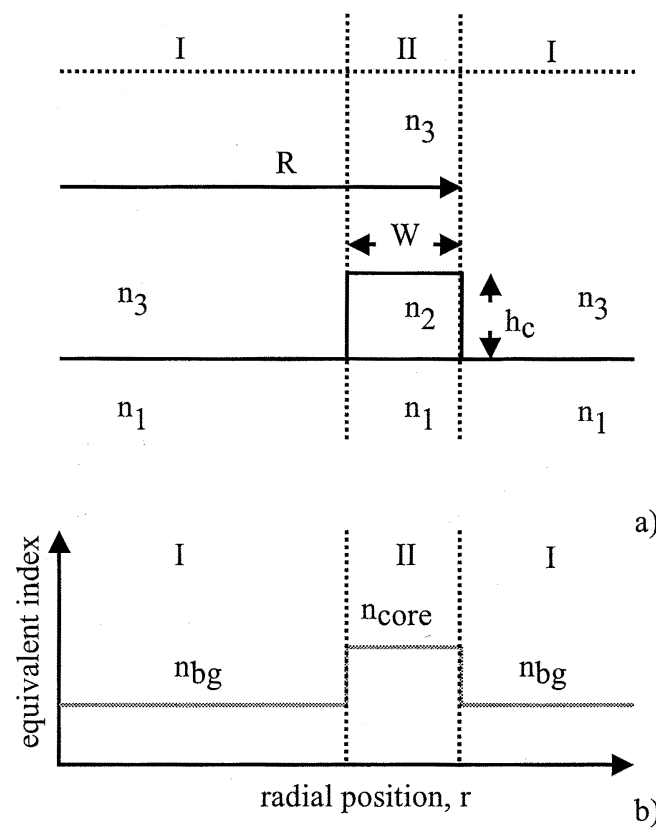

Fig. 5. (a) Vertical cross-section of a 3-D micro ring resonator with a radius of curvature $R$; a core with index of refraction $\mathrm{n}_{2}$, width $W$, and height $\mathrm{h}_{\mathrm{c}}$ on a semi-infinite buffer layer with index of refraction $\mathbf{n}_{1}$ and with a semi-infinite cladding with index of refraction $n_{3}$. (b) Radial index distribution of the equivalent 2-D micro ring resonator with an equivalent index of the core (II) and background (I) regions of $n_{\text {core }}$ and $n_{b g}$, respectively.

3-D system to a 2-D system with an equivalent radial index distribution; see Fig. 5(b). By applying the EIM to the vertical cross-section in the core region (II), one finds an equivalent index $\mathrm{n}_{\text {core }}$. The vertical cross-section in the background region (I) does not support a guided mode, and as a consequence, the EIM cannot be applied. As in most cases one is interested in an upper limit for the bend losses, the equivalent index of the background region is set to the maximum index of the background region: $\mathrm{n}_{\mathrm{bg}}=\max \left\{\mathrm{n}_{1}, \mathrm{n}_{3}\right\}$. Parameters like the bend losses and free spectral range of the 3-D micro ring resonator can be estimated by applying the analysis of Section III to the thus-obtained equivalent 2-D micro ring resonator. 
TABLE I

COMPARISON BETWEEN THE ROUND-TRIP BEND LOSSES OF THE FUNDAMENTAL TE POLARIZED MODES OF THE MiCRO RING RESONATOR IN FIG. 5 (WITH $\mathbf{n}_{1}=1.467, \mathbf{n}_{2}=1.997, \mathbf{n}_{3}=1.33$, AND $\lambda=780 \mathrm{~nm}$ ) ObTaINED by Using a 3-D Finite DifFERENCE MOde SOlver (3-D FD) AND THE NORMALIZEd ANALYSIS PROPOSED IN THIS PAPER (NORMALIZED)

\begin{tabular}{l|l|l|l|l}
\cline { 2 - 5 } & \multicolumn{2}{c|}{ 3D FD } & \multicolumn{3}{c}{ Normalized } \\
\hline $\mathbf{h}_{\mathbf{c}}[\mathbf{n m}]$ & $\boldsymbol{\alpha}_{\mathbf{r t r p}}[\mathbf{d B}]$ & $\mathbf{n}_{\text {core }}^{\prime}$ & $\boldsymbol{\alpha}_{\mathbf{r t r p}}[\mathbf{d B}]$ & $\mathbf{W}_{\text {WGM }}[\boldsymbol{\mu m}]$ \\
\hline 100 & $1.4 \cdot 10^{-1}$ & 1.067 & 2.6 & 1.9 \\
\hline 110 & $1.1 \cdot 10^{-2}$ & 1.081 & $3.4 \cdot 10^{-1}$ & 1.9 \\
\hline 120 & $7.5 \cdot 10^{-4}$ & 1.094 & $3.3 \cdot 10^{-2}$ & 1.9 \\
\hline
\end{tabular}

As a first example, consider a micro ring resonator-with a vertical cross-section like in Fig. 5(a)-defined in an antisymmetrical layer stack (PECVD $\mathrm{SiO}_{2}: \mathrm{n}_{1}=1.467$; LPCVD $\mathrm{Si}_{3} \mathrm{~N}_{4}: \mathrm{n}_{2}=1.997$, water: $\mathrm{n}_{3}=1.33$ ), radius of curvature $R=25 \mu \mathrm{m}$, ring width $W=2.5 \mu \mathrm{m}$, and operating at a wavelength of $\lambda=780 \mathrm{~nm}$. Table I shows the calculated bend losses per round-trip for the fundamental TE polarized mode obtained by applying a 3-D FD mode solver (3-D FD) and by applying the normalized analysis of Section III (normalized). From Table I, it can be concluded that the normalized analysis results in bend losses per round-trip about one order of magnitude larger than the bend losses per round-trip obtained by applying the 3-D FD mode solver. This is attributed to the chosen equivalent background index $\left(\mathrm{n}_{\mathrm{bg}}=1.467\right)$, which results in an underestimated lateral contrast and - as a consequence - in overestimated bend losses. As the ring width is sufficiently large ( $\left.W \geq W_{\mathrm{WGM}}\right)$ (see Table I), the approximation of the micro ring resonators by a micro disk is valid.

Next consider a micro ring resonator-with a vertical crosssection like in Fig. 5(a)—defined in a symmetrical layer stack $\left(\right.$ PECVD SiO $2: \mathrm{n}_{1}=1.465 ; \mathrm{LPCVD} \mathrm{Si}_{3} \mathrm{~N}_{4}: \mathrm{n}_{2}=1.979$, PECVD $\mathrm{SiO}_{2}: \mathrm{n}_{3}=1.465$ ), radius of curvature $R=25 \mu \mathrm{m}$, ring width $W=2.5 \mu \mathrm{m}$, and operating at a wavelength of $\lambda=1540 \mathrm{~nm}$. From Table II, it can be concluded that the normalized analysis-like in the previous example-overestimates the bend losses of the fundamental TE polarized (TE0,0) mode by one order of magnitude. The normalized analysis overestimates the free spectral range of the TE0,0 mode (see Table II) by $15 \%$, which is attributed to the fact that the normalized analysis does not account for waveguide and materials dispersion; see also (11). Even though the normalized graphs (Figs. 3 and 4) are calculated for the TM0 mode of a 2-D micro disk resonator-which corresponds with the TE polarized modes of the 3-D structure-they can also be used for estimating the parameters of the TM0,0 mode; Table II shows that also for this case, the normalized analysis gives reasonable estimates for the bend losses and the free spectral range. It should be remarked that even though for this example the fundamental modes are outside the WGM-regime (i.e., $W \leq W_{\mathrm{WGM}}$ ) - and as a consequence the micro ring resonator strictly spoken cannot be approximated by a micro disk resonator - the results of the normalized analysis agree reasonably well with the results obtained by the 3-D FD mode solver. This demonstrates that the WGM regime for the ring width as defined in (12) should be considered as indicative.

From the examples above, it can be concluded that by applying the normalized analysis of Section III to an equivalent
2-D micro disk resonator, reasonably accurate estimates for the bend losses and free spectral ranges of both the TE and TM polarized modes of a 3-D micro ring resonator can be obtained. The accuracy of the method is mainly limited by the reduction of the 3-D structure to an equivalent 2-D structure.

\section{Minimum SizE FOR 3-D MiCRORESONATORS}

In Section III, it has been shown that the minimum radius of curvature of a 2-D curved waveguide for given acceptable bend losses is determined by the available ratio between the index of the core and background. In this section, the minimum size (for given acceptable bend losses) for 3-D microresonators, with only a single mode order in the vertical ( $y$ ) direction, is investigated. Throughout this section, channel waveguides (index of guiding layer $\mathrm{n}_{1}$ ) of height $\mathrm{h}_{1}$ and core width $W \leq W_{\mathrm{WGM}}$ embedded in a uniform layer (index $n_{0}$ ) are assumed. Furthermore, the discussion is limited to the fundamental TE polarized (TE0,0) mode, and it is assumed-like in the previous section - that the 3-D microresonator can be reduced to a 2-D microresonator by applying the EIM for the vertical cross-section of the core region $\left(n_{1} \rightarrow n_{\text {core }}\right)$ and setting the background index of the resulting 1-D cross-section to $n_{b g}=n_{0}$. As a consequence, the TE0,0 mode of the 3-D microresonator becomes the TM0 mode of the 2-D microresonator.

By setting $h_{1}$ to the cutoff thickness of the first-order TE polarized slab mode $\left(h_{1}^{(\mathrm{TE} 1)}\right)$, it is possible to find an expression for the normalized core index $\mathrm{n}_{\text {core }}^{\prime}=\mathrm{n}_{\text {core }} / \mathrm{n}_{\mathrm{bg}}$ corresponding to the 1-D cross-section in terms of the index ratio $n_{1} / n_{0}$ of the 2-D cross-section. For a symmetric slab waveguide with an index ratio $\mathrm{n}_{1}^{\prime}=\mathrm{n}_{1} / \mathrm{n}_{0}$ and normalized-see (7) - core height $\mathrm{h}_{1}^{\prime}$, it is possible to express the well-known [13] normalized frequency $V$ and normalized guide index $b$ as

$$
\begin{aligned}
V & \equiv \frac{2 \pi}{\lambda} \mathrm{h}_{1} \sqrt{\mathrm{n}_{1}^{2}-\mathrm{n}_{0}^{2}}=2 \pi \mathrm{h}_{1}^{\prime} \sqrt{\mathrm{n}_{1}^{\prime 2}-1} \\
\mathrm{~b} & \equiv \frac{\left(\mathrm{n}_{\text {core }}^{\prime}\right)^{2}-1}{\left(\mathrm{n}_{1}^{\prime}\right)^{2}-1} .
\end{aligned}
$$

From the $V$-parameter in (13), it is straightforward to derive the normalized cutoff thickness of the TE1 slab mode

$$
\begin{aligned}
& V^{(\mathrm{TE} 1)}=\pi=2 \pi \mathrm{h}_{1}^{\prime} \sqrt{\mathrm{n}_{1}^{\prime 2}-1} \\
& \mathrm{~h}_{1}^{\prime(\mathrm{TE} 1)}=\frac{1}{2 \sqrt{\mathrm{n}_{1}^{\prime 2}-1}} .
\end{aligned}
$$

In this case, the dispersion relation for the fundamental TE polarized slab mode becomes [13]

$$
\frac{\pi \sqrt{1-\mathrm{b}^{(\mathrm{TE} 0)}}}{2}=\tan ^{-1}\left(\sqrt{\frac{\mathrm{b}^{(\mathrm{TE} 0)}}{1-\mathrm{b}^{(\mathrm{TE} 0)}}}\right) \text {. }
$$

This transcendental equation can be solved numerically

$$
\mathrm{b}^{(\mathrm{TE} 0)} \sim 0.646
$$

Upon insertion of (16) into (13), it is possible to express $n_{\text {core }}^{\prime}$ (corresponding to the cutoff height of the core region) in terms of $\mathrm{n}_{1}^{\prime}$

$$
\mathrm{n}_{\text {core }}^{\prime}=\sqrt{1+\left(\left(\mathrm{n}_{1}^{\prime}\right)^{2}-1\right) \cdot \mathrm{b}^{(\mathrm{TE} 0)}} .
$$


TABLE II

COMPARISON BETWEen THE Round-TRIP BEND LOSSES $\left(\alpha_{\mathrm{rtrp}}\right)$ AND FSRS OF THE FUNDAMENTAL MOdES OF THE MicRo RING RESONATOR IN FIG. 5 (WITH $\mathrm{n}_{1}=1.465, \mathrm{n}_{2}=1.979, \mathrm{n}_{3}=1.465$, AND $\lambda=1540 \mathrm{~nm}$ ) OBTAINED By Using A 3-D FD Mode SOlver AND THE NORMALIZED ANALYSIS PROPOSED IN

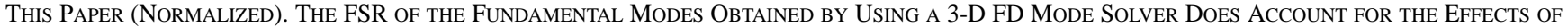

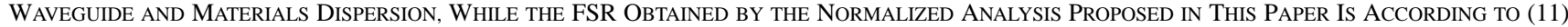
AND EXCLUDES THE EFFECTS OF WAVEGUIDE AND MATERIALS DISPERSION

\begin{tabular}{l|l|l|l|l|l|l}
\cline { 2 - 7 } & \multicolumn{2}{c|}{ 3D FD } & \multicolumn{4}{c}{ Normalized } \\
\hline Mode & $\boldsymbol{\alpha}_{\text {rtrp }}[\mathbf{d B}]$ & FSR $[\mathbf{n m}]$ & $\mathbf{n}_{\text {core }}^{\prime}$ & $\boldsymbol{\alpha}_{\text {rtrp }}[\mathbf{d B}]$ & FSR $[\mathbf{n m}]$ & $\mathbf{W}_{\text {WGM }}[\boldsymbol{\mu m}]$ \\
\hline TE0,0 & $4.8 \cdot 10^{-3}$ & 8.2 & 1.146 & $3.8 \cdot 10^{-2}$ & 9.4 & 3.0 \\
\hline TM0,0 & 3.9 & 8.8 & 1.082 & 12 & 9.9 & 2.9 \\
\hline
\end{tabular}

Using the thus-found value for $\mathrm{n}_{\text {core }}^{\prime}$ and the normalized radius of curvature $R^{\prime}$, it is now straightforward to find the losses per round-trip from Fig. 3.

As an example, consider an MR made in silicon oxynitride (SiON) technology whose fundamental TE polarized mode has an unloaded finesse-considering only the bend losses-of at least $F^{(0)} \geq 2.7 \cdot 10^{3}$ (i.e., $\alpha_{\text {rtrp }} \leq 0.01 \mathrm{~dB}$ ). For SiON layers, the index can be varied between the index of $\mathrm{SiO}_{2}(\mathrm{n} \sim 1.45)$ and $\mathrm{Si}_{3} \mathrm{~N}_{4}(\mathrm{n} \sim 2.0)$ [12], and as a consequence, the ratio between the (effective) indexes and the height of the core region is limited to

$$
1 \leq \mathrm{n}_{1}^{\prime} \leq 1.38:\left\{\begin{array}{l}
\mathrm{n}_{\text {core }}^{\prime} \leq 1.26 \\
\mathrm{~h}_{1}^{\prime} \leq 0.53
\end{array}\right.
$$

For an MR embedded in a $\mathrm{SiO}_{2}$ layer with a $\mathrm{Si}_{3} \mathrm{~N}_{4}$ guiding layer (i.e., $\mathrm{n}_{1}^{\prime}=1.38$ ) and only a single mode in the vertical (y) direction (i.e., $\mathrm{h}_{1}^{\prime}=0.53$ ), it can be inferred from Fig. 3 that the normalized radius should be $R^{\prime} \geq 12$. For wavelengths in the third telecom window (i.e., around $\lambda=1.55 \mu \mathrm{m}$ ), this corresponds with an $\mathrm{h}_{1} \sim 0.57 \mu$ m-thick $\mathrm{Si}_{3} \mathrm{~N}_{4}$ layer, radius of curvature of $R \geq 12.8 \mu \mathrm{m}$, and a free spectral range $\leq 18 \mathrm{~nm}$.

\section{CONCLUSION}

Normalized graphs of the bend losses, unloaded finesse, and free spectral range of the fundamental quasiguided modes in a cylindrical microresonator have been presented. It has been demonstrated that, by using these normalized graphs, it is possible to rapidly estimate the performance of a 2-D cylindrical microresonator (for both polarizations) with only the normalized radius of curvature and the ratio between the indexes of the core and background layers as input. By reducing a 3-D microresonator to a 2-D microresonator and applying the normalized graphs to the equivalent 2-D microresonators, reasonably accurate estimates for parameters like the free spectral range and bend losses of the fundamental modes of 3-D microresonators could be obtained. The minimum size-for given acceptable bend losses and ratio between the indexes of the guiding and background layers-for 3-D microresonators embedded in a uniform layer could be estimated. The method presented here does not require detailed numerical simulations and can considerably speed up the design of cylindrical microresonators.

\section{REFERENCES}

[1] B. E. Little, S. T. Chu, H. A. Haus, J. Foresi, and J. P. Laine, "Microring resonator channel dropping filters," J. Lightwave Technol., vol. 15, pp. 998-1005, 1997.
[2] D. J. W. Klunder, E. Krioukov, F. S. Tan, T. van der Veen, H. F. Bulthuis, G. Sengo, C. Otto, H. J. W. M. Hoekstra, and A. Driessen, "Vertically and laterally waveguide-coupled cylindrical microresonators in $\mathrm{Si}_{3} \mathrm{~N}_{4}$ on $\mathrm{SiO}_{2}$ technology," Appl. Phys. B, vol. 73, pp. 603-608, 2001.

[3] D. J. W. Klunder, M. L. M. Balistreri, F. C. Blom, H. J. W. M. Hoekstra, A. Driessen, L. Kuipers, and N. F. van Hulst, "Detailed analysis of the intracavity phenomena inside a cylindrical microresonators," J. Lightwave Technol., vol. 20, pp. 519-529, 2002.

[4] W. W. Lui, C.-L. Xu, T. Hirono, K. Tokoyama, and W.-P. Huang, "Full vectorial wave propagation in semiconductor optical bending waveguides and equivalent straight waveguide approximations," J. Lightwave Technol., vol. 16, pp. 910-914, 1998.

[5] Commercially available finite-difference mode solvers for bends with 1D and 2D cross-sections that are an integrated part of 'Olympios,' Concept to Volume (C2V), Enschede, The Netherlands.

[6] E. C. M. Pennings, "Bends in Optical Ridge Waveguides: Modeling and Experiments," PhD thesis, University of Delft, Delft, The Netherlands, 1990.

[7] M. K. Smit, E. C. M. Pennings, and H. Blok, "A normalized approach to the design of low-loss optical waveguide bends," J. Lightwave Technol., vol. 11, pp. 1737-1742, 1993.

[8] J. R. Reitz, Foundations of Electromagnetic Theory, 4th ed. Reading, MA: Addison-Wesley, 1993.

[9] D. Marcusse, "Light transmission optics," in Van Nostrand Reinhold Electrical/Computer Science and Engineering Series, 2nd ed. New York, 1982.

[10] S. C. Hagness, D. Rafizadeh, S. T. Ho, and A. Taflove, "FDTD microcavity simulations: design and experimental realization of waveguide-coupled single-mode ring and whispering-gallery-mode disk resonators," J. Lightwave Technol., vol. 15, pp. 2154-2165, 1997.

[11] W. P. Huang, C. L. Xu, W. Lui, and K. Yokoyama, "The perfectly matched layer boundary condition for modal analysis of optical waveguides: leaky mode calculations," IEEE Photon. Technol. Lett., vol. 8, pp. 652-654, 1996.

[12] K. Wörhoff, P. V. Lambeck, and A. Driessen, "Design, tolerance analysis, and fabrication of silicon oxynitride based planar optical waveguides for communication devices," J. Lightwave Technol., vol. 17, pp. 1401-1407, 1999.

[13] H. Kogelnik and V. Ramaswamy, "Scaling rules for thin-film waveguide," Appl. Opt., vol. 13, pp. 1857-1862, 1974.

Dion J. W. Klunder (S'98-M'02) was born in The Netherlands in 1973. He received the M.Sc. and Ph.D. degrees in applied physics (integrated optics) from the University of Twente, Enschede, The Netherlands.

He is now with Concept to Volume b.v., Enschede, The Netherlands, where he is a Design Engineer. His interest is focused on integrated optics and microtechnology in general.

H. J. W. M. Hoekstra, photograph and biography not available at the time of publication.

Alfred Driessen (M'93-SM'95) received the Ph.D. degree in solid-state physics from the University of Amsterdam, The Netherlands, in 1982.

After completing his doctoral work, he was a Postdoctoral Researcher on metal hybrides with the Free University of Amsterdam. In 1988, he joined the Lightwave Devices Group, University of Twente, Enschede, The Netherlands, as an Associate Professor. Presently his interest focuses on compact photonic structures, such as microresonators and photonic wires that eventually could lead to very large scale integrated (VLSI) photonics. 